\title{
Mapping the Left Ventricular Cavity by Analysis of Images Reconstructed from 3D Trans-oesophageal Echocardiography
}

\author{
AJ Sims ${ }^{1}$, J Pemberton $^{2}$, T Irvine ${ }^{2}$, A Kenny $^{2}$, A Murray $^{1}$ \\ ${ }^{1}$ Regional Medical Physics Department, Freeman Hospital, Newcastle upon Tyne, UK \\ ${ }^{2}$ Department of Cardiology, Freeman Hospital, Newcastle upon Tyne, UK
}

\begin{abstract}
Three dimensional measurement of the left ventricle (LV) has many clinical uses but is difficult to achieve. We present a new technique for automatic mapping of the LV cavity using images reconstructed from $3 D$ transoesophageal echocardiography.

$3 D$ image sets were constructed from an angular sequence of conventional B-mode frames. Reconstructed $2 D$ slices perpendicular to the $L V$ axis were analysed to find the boundary of the LV cavity in each slice. 3D maps of the cavity were constructed by combining boundary positions from successive slices. Images from 3 patients were used to test the algorithm. Consistency was measured by analysing slice-to-slice differences in the boundary position and accuracy was measured by comparison with manual measurements by a cardiologist.

Results of this preliminary study show that reconstruction and analysis of images perpendicular to the LV axis is a practical technique for mapping the LV cavity.
\end{abstract}

\section{Introduction}

Three-dimensional measurement of the left ventricle (LV) by echocardiography has many clinical uses but is difficult to achieve $[1,2]$. Clinical studies have confirmed that LV volume measurements by echocardiography give comparable results to those obtained with other modalities. For example, Mele et al [3] constructed wireframe LV maps from 2D trans-thoracic images by manually tracing endocardial borders in patients and normal subjects and showed that calculated end-diastolic (ED) and end-systolic (ES) volume, stroke volume and ejection fraction were in good agreement with results from other independent imaging techniques. In children with congenital heart defects, Heusch et al [4] analysed slices from 3D transoesophageal echocardiography using manual planimetry and found that LV volumes were correlated with, but smaller than those calculated from angiocardiography.

Manual identification of the endocardial border is time consuming, but several studies report research into automatic boundary detection. Sonka et al [5] acquired images of dog and pig hearts using intracardiac echocardiography and found that LV cavity areas and volumes based on automatic detection of borders using graph searching agreed well with manual measurements. Stetten et al [6] used fuzzy labelling of voxels in real time 3D trans-thoracic echocardiography data to locate the left ventricular axis and cavity walls and showed good agreement with manual tracings. Taking a different approach, Rodevand et al [7] compared various multiplane 3D echocardiographic methods using asymmetric latex phantoms and achieved best results when the probe was directed perpendicularly to the ventricular long axis.

In this paper, a new technique is presented for the automatic detection of LV walls from synthesised images perpendicular to the LV axis reconstructed from 3D transoesophageal echocardiography (TOE) studies.

\section{Methods}

\subsection{Image capture}

TOE studies were carried out on patients who had undergone coronary artery bypass graft surgery. All had impaired LV function. Images were acquired in B-mode using an ATL HDI 5000 ultrasound system with a $7 \mathrm{MHz}$ MPT 7-4 probe whilst ventilation was suspended. A sequence of frames over one cardiac cycle was captured for each orientation of the transducer. The image plane was progressively rotated at $6^{\circ}$ intervals about an axis parallel to the LV axis to establish a 3D image set. The number of frames per R-R interval was constant for each patient and depended upon heart rate.

\subsection{Image analysis}

For each study analysed, 30 frames (1 for each orientation) from end-diastole were combined to form a 3D image set using specially written software. Similar sets were constructed from end-systolic frames. The software permitted 2D images to be extracted from these 


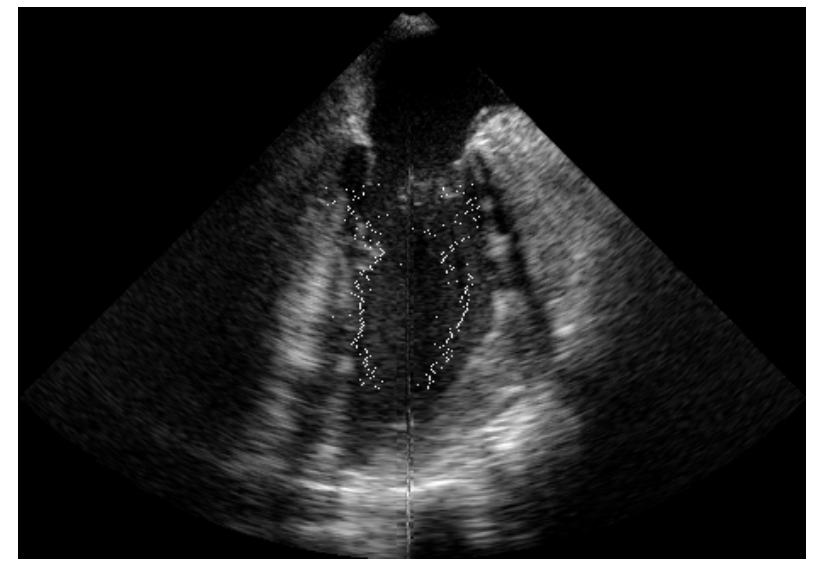

Figure 1. Planar image reconstructed from 3D study for patient 1 at ED. Bright white spots show the projections of the automatically calculated LV wall.
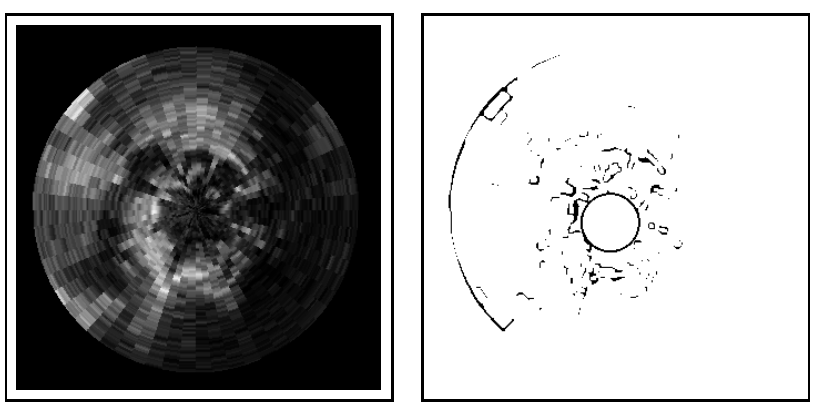

Figure 2. Left: Planar image reconstructed perpendicularly to the beam axis for patient 1 at ES approximately mid-way between the apex and the mitral valve. Right: The same image following application of edge detection and thresholding operators.

3D image sets, where the reconstructed image plane was either parallel or perpendicular to the beam axis. Figure 1 shows a reconstructed image with plane parallel to the beam axis for one patient at a beam orientation of $0^{\circ}$; this is equivalent to one of the original B-mode source frames.

100 reconstructed 2D images were created from each image set in planes perpendicular to the $\mathrm{LV}$ axis extending from just above the apex to just below the mitral valve. An example is shown in the left hand panel of figure 2 . These images were automatically processed using edge detection, thresholding and morphological operators (see, for example Pratt [8]) to create binary edge maps (figure 2, right hand panel). Within each edge map, the LV cavity was modelled as the largest circle which included the beam axis, but which did not touch any surrounding tissue structure. The best fit LV cavity for the image on the left of figure 2 is shown in the panel on the right.

Circles modelling local parts of the LV were combined to form 3D maps. These maps were projected onto original

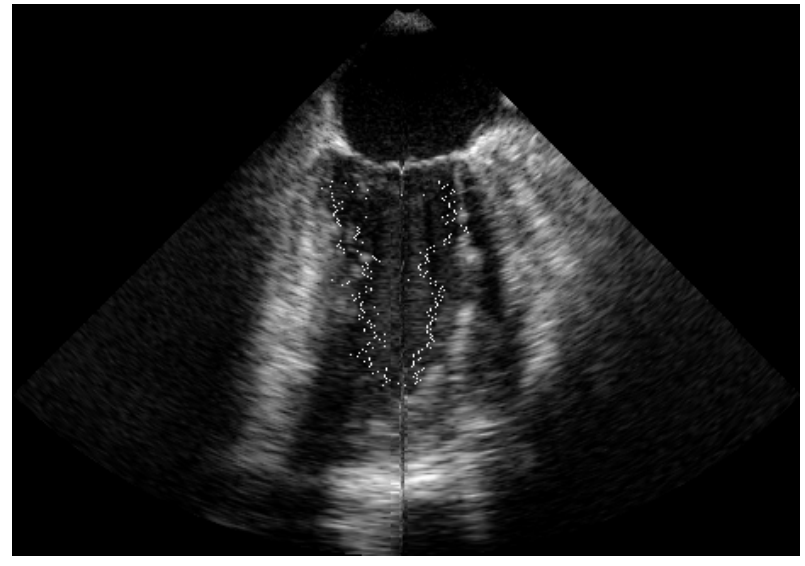

Figure 3. Planar image reconstructed from 3D study for patient 1 at ES. The image plane is the same as that for figure 1 and the bright white spots are the projections of the automatically calculated LV wall.

B-mode frames in order to permit quantitative assessment of the technique. Projections taken from maps at different parts of the cardiac cycle are shown in figures 1 and 3 .

\subsection{LV cavity measurements}

Since each slice was calculated independently, the consistency of the algorithm was assessed by measuring slice-to-slice differences of the radius and position of the centre of each circular fit to the LV wall for all 100 slices.

LV cavity dimensions (ie the distance between walls perpendicular to the beam axis) were measured manually by a cardiologist for 2 depths, 4 orientations and 2 phases of the cardiac cycle for each patient, and were compared to projections of the LV map for each study using the method of Bland and Altman [9].

\section{Results}

\subsection{Slice-to-slice variations}

Table 1 shows the mean slice-slice variations of the radius of the LV cavity and the position of the $L V$ axis (in a plane perpendicular to the LV axis).

The radius and position of the LV map in each slice were expected to vary smoothly along the LV axis. Figure 4 shows the variation of these two quantities for one patient at ES. 
Table 1. Mean slice-to-slice variations for the local radius ( $r$ ) of the LV cavity, and for the position of the LV long axis (c). All distances are in $\mathrm{mm}$.

\begin{tabular}{cccccc}
\hline & \multicolumn{2}{c}{ ED } & & \multicolumn{2}{c}{ ES } \\
\cline { 2 - 3 } \cline { 5 - 6 } Patient & $\mathrm{r}$ & $\mathrm{c}$ & & $\mathrm{r}$ & $\mathrm{c}$ \\
\hline 1 & 0.9 & 2.2 & & 0.9 & 1.4 \\
2 & 1.1 & 2.0 & & 1.5 & 2.2 \\
3 & 2.6 & 3.8 & & 2.0 & 2.5 \\
\hline
\end{tabular}

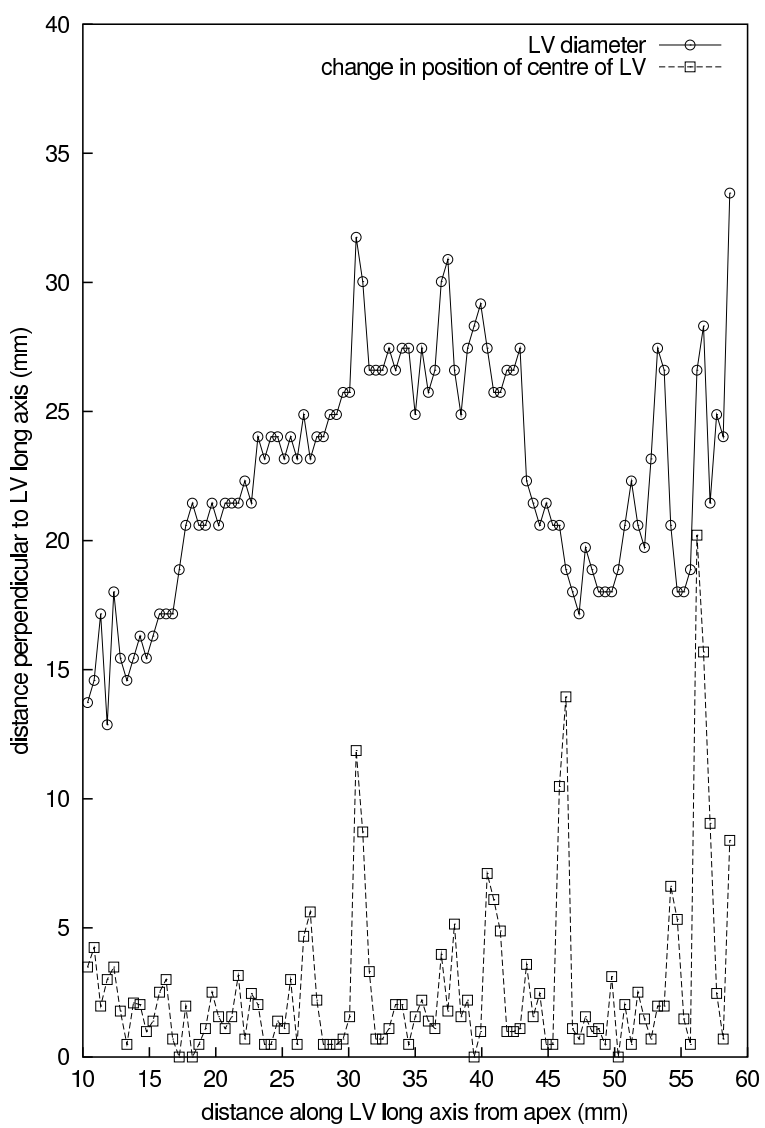

Figure 4. Slice-to-slice variation of LV diameter (upper curve) and change in position of LV long axis for patient 1 (lower curve). Smooth changes in both quantities were expected, and spikes indicate slices where the estimated LV wall position has a higher degree of uncertainty.
Table 2. Comparison of automatic and manual measurements of left ventricular cavity wall-to-wall distance for patient 2. Each measurement was perpendicular to the LV long axis. There were 16 separate comparisons ( 2 beam depths $\times 4$ angles $\times 2$ phases of the cardiac cycle).

\begin{tabular}{rrrrrr}
\hline \multirow{2}{*}{$\begin{array}{c}\text { depth } \\
(\mathrm{mm})\end{array}$} & $\begin{array}{c}\text { angle } \\
\text { (degrees) }\end{array}$ & phase & \multicolumn{3}{c}{ LV cavity dimension $(\mathrm{mm})$} \\
\cline { 4 - 6 } 101 & 0 & ED & 19.7 & 25.0 & -5.3 \\
101 & 0 & ES & 6.8 & 3.4 & 3.4 \\
101 & 42 & ED & 18.1 & 20.6 & -2.5 \\
101 & 42 & ES & 5.4 & 6.9 & -1.5 \\
101 & 90 & ED & 15.8 & 15.7 & 0.1 \\
101 & 90 & ES & 6.6 & 11.3 & -4.7 \\
101 & 132 & ED & 17.3 & 14.2 & 3.0 \\
101 & 132 & ES & 6.7 & 0.0 & 6.7 \\
71 & 0 & ED & 13.2 & 32.4 & -19.2 \\
71 & 0 & ES & 22.5 & 24.5 & -2.1 \\
71 & 42 & ED & 13.3 & 24.5 & -11.3 \\
71 & 42 & ES & 22.5 & 25.5 & -3.0 \\
71 & 90 & ED & 13.8 & 21.6 & -7.8 \\
71 & 90 & ES & 22.5 & 17.7 & 4.8 \\
71 & 132 & ED & 13.6 & 19.6 & -6.0 \\
71 & 132 & ES & 22.7 & 27.5 & -4.9 \\
\hline
\end{tabular}

\subsection{Comparison with measurement by cardiologist}

Table 2 shows the results of 16 separate comparisons between automatic and manual measurements for one patient. The mean difference (automatic-manual) between the techniques was $-3.1 \mathrm{~mm}(95 \%$ confidence interval -6.6 to $0.3 \mathrm{~mm}$ ). In the table, depth refers to distance from the beam source; $101 \mathrm{~mm}$ depth corresponds to approximately $40 \mathrm{~mm}$ from the apex, and $71 \mathrm{~mm}$ depth corresponds to approximately $100 \mathrm{~mm}$ from the apex.

Table 3 shows the mean differences between automatic and manual measurements for the 16 individual comparisons for each patient. In all cases, the automatic technique identifies closer boundaries, although this is significant at the $95 \%$ confidence level in only one case.

\section{Discussion}

Two dimensional echocardiographic images of the left ventricle are sections through the vessel and by definition contain only a fraction of the information needed to calculate volume. However, clinical research studies have shown that $3 \mathrm{D}$ echocardiographic images can be used to measure physical characteristics of the LV including volume. 
Table 3. Left ventricular cavity wall-to-wall distance measured by the automatic technique compared to manual measurement by a cardiologist. The method of Bland and Altman was used to compare sixteen separate measurements for each patient. Differences are automatic - manual.

\begin{tabular}{rrc}
\hline Patient & bias (mm) & 95\% CI of bias \\
\hline 1 & -8.2 & -11.8 to -4.7 \\
2 & -3.1 & -6.6 to 0.3 \\
3 & -4.5 & -9.0 to 0.1 \\
\hline
\end{tabular}

Conventional 2D echocardiography is limited by anatomical constraints to a restricted set of views. In addition, 2D images often contain irregular boundary reflections and shadowing effects, making automatic detection of boundaries difficult. In contrast, threedimensional reconstruction permits images to be synthesised for arbitrary viewpoints and provides opportunity for improved border detection based on 3D algorithms.

In this preliminary study we have demonstrated that reconstruction and analysis of images perpendicular to the LV axis from source images obtained by trans-oesophageal echocardiography is a practical technique for mapping the LV cavity.

Further improvement of the method is possible. The present LV wall detection algorithm uses information from a single slice perpendicular to the LV axis. This can be improved by making use of binary edge points in neighbouring slices based on the assumption of local smoothness of the shape of the vessel. Finally, the method should be validated by comparing automatically calculated volume against an independent means of measurement.

\section{References}

[1] Fenster A, Downey D, Cardinal H. Three dimensional ultrasound imaging. Physics in Medicine and Biology 2001; 46:R67-R99.

[2] Frangi A, Niessen W, Viergever M. Three-dimensional modelling for functional analysis of cardiac images: A review. IEEE Transactions on Medical Imaging 2001;20:225.

[3] Mele D, Maehle J, Pedini I, Alboni P, Levine R. Threedimensional echocardiographic reconstruction: decription and applications of a simplified technique for quantitative assessment of left ventricular size and function. American Journal of Cardiology 1998;81:107G-110G.

[4] Heusch A, Rubo J, Krogmann O, Bonig H, Bourgeois M. Volume measurement of the left ventricle in children with congenital heart defects: 3-dimensional echocardiography versus angiocardiography. Cardiology 1999;92:45-52.

[5] Sonka M, Liang WD, Kanani P, Allan J, DeJong S, Kerber $\mathrm{R}, \mathrm{McKay} \mathrm{C}$. Intracardiac echocardiography: computerized detection of left ventricular borders. International Journal of Cardiac Imaging 1998;14:397-411.

[6] Stetten G, Pizer S. Medial-guided fuzzy segmentation. Lecture Notes in Computer Science 2000;1935:226-35.

[7] Rodevand O, Bjornerheim R, Aakhus S, Kjekshus J. Left ventricular volumes assessed by different new three-dimensional echocardiographic methods and ordinary biplane technique. International Journal of Cardiac Imaging 1998;14:55-63.

[8] Pratt W. Digital Image Processing. Second edition. New York, USA: John Wiley \& Sons Inc, 1991.

[9] Bland JM, Altman D. Statistical methods for assessing agreement between two methods of clinical measurement. Lancet 1986;1 (8476):307-310.

Address for correspondence:

Andrew J Sims

Regional Medical Physics Department, Freeman Hospital

Newcastle upon Tyne, UK.

tel.: +441912843111 ×26598

a.j.sims@newcastle.ac.uk 